\title{
Two cases of severe pneumonia after the 2011 Great East Japan Earthquake
}

\author{
Toshihide Nakadate, ${ }^{a}$ Yutaka Nakamura, ${ }^{a}$ Kohei Yamauchii ${ }^{a}$ and Shigeatu Endoa \\ Correspondences to Toshihide Nakadate (e-mail: tnakadat@iwate-med.ac.jp).
}

In 2011, during the Great East Japan Earthquake and tsunami, 90\% of victims died from drowning. We report on two tsunami survivors with severe pneumonia potentially caused by Legionella pneumophila. Both victims aspirated a large quantity of contaminated water; sand, mud and a variety of microbes were thought to have entered into their lower respiratory tracts. One patient had a mycotic intracranial aneurysm; the other patient had co-infections with several organisms, including Scedosporium species. Although scedosporiosis is a relatively rare infectious disease, symptoms are progressive and prognosis is poor. These pathogens are not specific for tsunami lung, but are reported causative agents for pneumonia after near-drowning.

$\mathrm{N}$ atural disasters can result in excess morbidity and mortality due to infectious diseases. Diseases that cause dramatic epidemics, such as measles, cholera, dysentery and malaria, are usually considered the main threats during humanitarian relief operations. Acute respiratory infections (ARI) have received far less attention in humanitarian relief and preparedness programmes despite recent evidence suggesting high excess morbidity and mortality and case fatality rates due to ARI occur during such events. ${ }^{1}$ After the initial rush of patients with injury after the Great HanshinAwaji earthquake in 1995, the number of respiratory diseases, largely pneumonia, increased about 4.5-fold. ${ }^{2}$

Tsunamis, which result from sudden changes in the seafloor, can occur after an earthquake and can result in large quantities of earth and sand being swept up and deposited, leaving behind sediment called tsunami deposit. This tsunami deposit, found at various places that the tsunami passes and near to shoreline, can infect the soil. After the Indian Ocean earthquake and tsunami in 2004, multidrug-resistant bacterial infections were often found in the survivors, and a tsunami-related tetanus epidemic was reported. ${ }^{3}$ Necrotizing pneumonia and pulmonary abscesses seen by the survivors of tsunamis were named tsunami lung. ${ }^{4,5}$

Tsunami lung occurs when people being swept by tsunami waves inhale salt-water contaminated with mud and bacteria. In some patients with tsunami lung, sand and plant fragments are collected from bronchoalveolar lavage fluid; therefore, the patients aspirate not only industrial materials but also various microbes that live in seawater, freshwater and soil. The resulting pneumonialike infections are normally treated with antibiotics. However, when medical infrastructure is destroyed in such events and antibiotics are not available to treat infections in the early stages, pulmonary infections can fester, enter the bloodstream and spread to the brain, producing abscesses.

On 11 March 2011, a major 9.0-magnitude earthquake was generated near Japan, and the tsunami that resulted engulfed the Pacific coast of the Tohoku district of Japan, causing serious damage. The Japanese National Police Agency reported that of the 15467 deaths, 13135 (92.4\%) were due to drowning.

We report on two cases of Legionnaires' disease that developed after near-drowning caused by this tsunami.

\section{CASE PRESENTATION}

\section{Case 1: 33-year-old women}

The patient was engulfed by the tsunami in Rikuzen-takada city, Iwate prefecture, Japan on 11 March 2011 and was rescued. Heavy oil covered her body. She was transported to the emergency centre with suspicion of chemical pneumonitis. Both lungs showed diffuse infiltrative shadows on a chest X-ray. Chemical pneumonitis from aspiration of heavy oil was diagnosed. Treatment included

Department of Critical Care Medicine, Department of Respiratory Medicine, Iwate Medical University, Uchimar 19-1, Morioka, Iwate, Japan. Submitted: 11 April 2012; Published: 30 October 2012

doi: 10.5365/wpsar.2012.3.2.002 
Figure 1. 33-year-old woman's chest X-ray showed alveolar infiltrate with air bronchogram in left upper lobe, and showed multifocal, bilateral nodular opacities

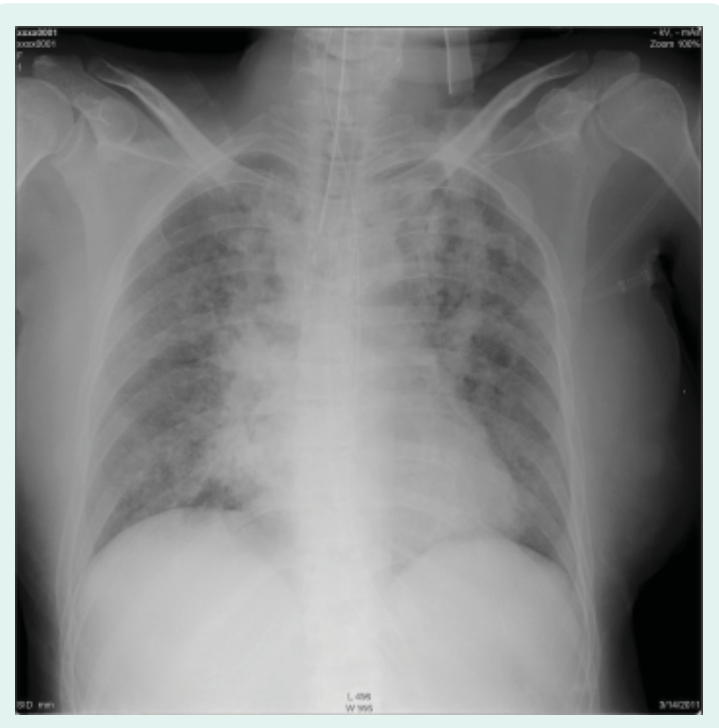

meropenem, administration of Sivelestat sodium and steroid pulse therapy.

In spite of administration of antibiotics, her respiratory state and oxygenation worsened. The chest $X$-ray showed alveolar infiltrates in the left upper lobe; it also showed multifocal, bilateral nodular opacities (Figure 1).

On day 17, massive haemoptysis occurred and because of airway occlusion and hypotension, the patient died. In the bronchoalveolar lavage fluid, Legionella pneumophila serogroup-1, Pseudomonas aeruginosa, Stenotrophomonas maltophilia, Burkholderia cepacia and filamentous fungi Scedosporium apiospermum and Scedosporium prolificans were detected. As a cause of massive haemoptyisis, necrotizing pneumonia and haemorrhagic infarct by these filamentous fungi were suspected.

\section{Case 2: two-year-old girl}

This patient was also engulfed in the tsunami in Rikuzen-takada city, Iwate prefecture, Japan on 11 March 2011 and was transported to our hospital on the following day, 12 March. We initially suspected aspiration pneumonia and started administration of ceftaxim. Because Pseudomonas aeruginosa was detected by sputum culture, we switched to piperacillin,
Figure 2. Two-year-old girl's chest X-ray showed right upper lobe consolidation and infiltrative shadow in left upper lobe

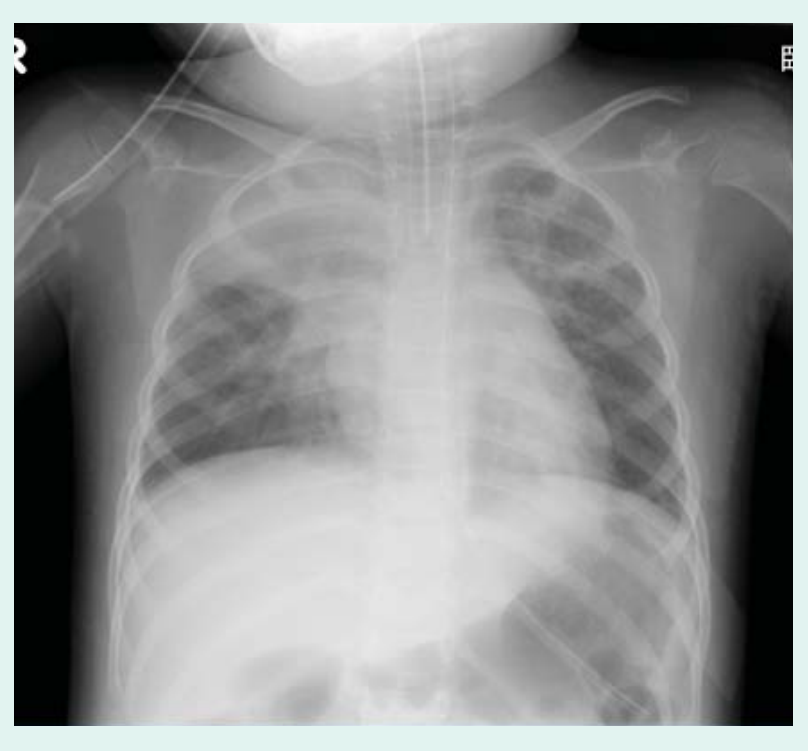

but the chest shadow worsened (Figure 2). We then suspected Legionnaires' disease. Results of a urinary antigen test was positive and her antibody titre for Legionella pneumophila rose to 1024-fold. After Legionnaires' disease was diagnosed, and levofloxacin administered, the shadow of the right superior lobe was improved according to a chest film.

Her symptoms improved gradually, but sudden conjugate deviation and tonic convulsions of both lower extremities developed on day 23. A computed tomography scan of her brain showed bleeding around the brainstem associated with subarachnoid haemorrhage and hydrocephalus (Figure 3). A mycotic aneurysm was considered as the cause of the brainstem haemorrhage. The patient died on the 36th day of illness.

\section{DISCUSSION}

This report documents the development of two cases of Legionnaires' disease after the Great East Japan Earthquake disaster. This is not the first report of this diagnosis after this event. ${ }^{6}$

Legionella species inhabit fresh water environments and have been detected in rivers and brackish water areas. Both patients with Legionnaires' disease were rescued in a neighbourhood near a river that was destroyed by the tsunami, and so it is possible that 
Figure 3. Computed tomography scan of the brain after convulsion shows bleeding around brainstem associated with subarachnoid haemorrhage and hydrocephalus

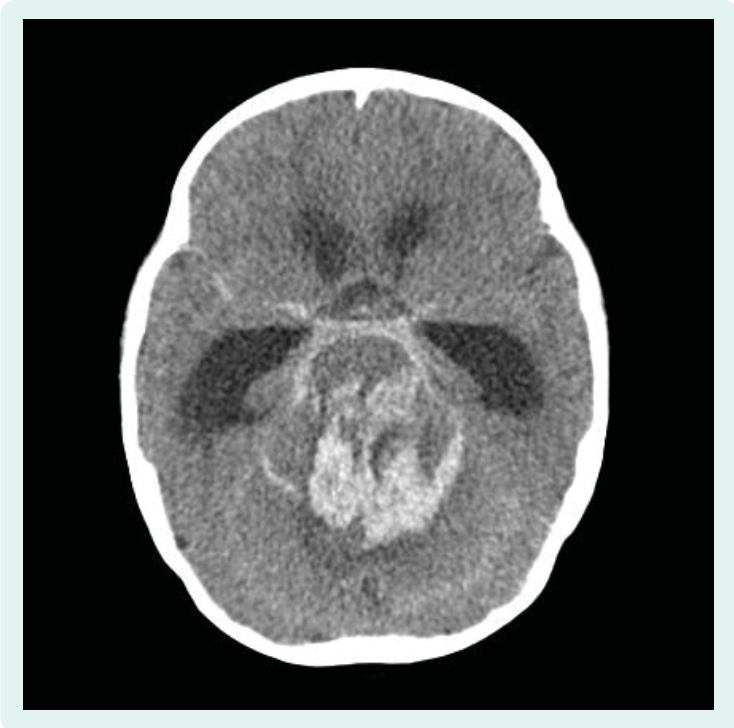

Legionella pneumophila may have been in the river and became part of the tsunami deposit.

Community-acquired pneumonia by Legionella pneumophila is extremely rare in Thailand, and in New Zealand and Australia, where Legionella longbeachae accounts for about half of Legionnaires' disease cases. ${ }^{7}$ This may be due to differences in the living environment as Legionella bacteria multiply in man-made environmental waters and often cause human infection. The identification of the source of infection is important when we consider the high mortality rate of Legionnaires' disease and the widespread man-made water environments. Blood-streaked phlegm or haemoptysis occurs in about one-third of Legionnaires' disease patients, but massive haemoptysis is not common. ${ }^{8}$ In contrast, cases of Legionella pneumophila pneumonia followed by invasive aspergillosis have been reported. ${ }^{9}$ Neurologic symptoms and neurologic manifestations are known to be often found in Legionnaires' disease. However, brain abscess or cerebral haemorrhage has not been reported until now, so it difficult to conclude that the cerebral haemorrhage of Case 2 was due to Legionnaires' disease.

As for the cause of the massive haemoptysis of Case 1, superinfection of filamentous fungi is suspected. The filamentous fungi Scedosporium apiospermum, as well as the gram-negative bacilli Pseudomonas aeruginosa, Stenotrophomonas maltophilia and Burkholderia cepacia were all detected in Case 1. These bacteria have the ability to produce $\beta$-lactamase and form biofilm and are therefore resistant to antibiotics such as carbapenem or penicillin. These bacteria and filamentous fungi are widespread in soil and fresh water.

Scedosporium apiospermum is particularly known to cause systemic invasive mycosis after near-drowning. Apiospermum is resistant to many antifungal agents, and only voriconazole shows susceptibility in the available antifungal agents. In a review of 22 Scedosporium apiospermum infections that occurred after neardrowning, dissemination to the central nervous system was confirmed at high frequency $(91 \%)$, with the most common lesion being multiple brain abscesses $(65 \%) .{ }^{10}$ The same study reported that the onset of symptoms can be slow with a delayed time to diagnosis of up to 28 days. ${ }^{10} \mathrm{~A}$ patient who developed an infectious cerebral aneurysm due to scedosporiosis after near-drowning has also been reported, ${ }^{11}$ and the mycotic contribution of Scedosporium spp. was suspected. In the East Japan tsunami, multiple brain abscesses by Scedosporium apiospermum also developed and were cured by antifungal treatment. ${ }^{12}$

General mycological staining does not discriminate Scedosporium and other filamentous fungi such as Aspergillus and Fusarium. Cultures of cerebrospinal fluid from infected patients may be negative or delayed up to three weeks. ${ }^{13}$ Other diagnostic tests previously reported are polymerase chain reaction and immuno-chromatographic lateral-flow device. ${ }^{14}$ During disasters, complicated instruments may not work due to power failure or shortage of reagents and materials. In New Orleans, after Hurricane Katrina in 2005, United States sea, land and airborne rescue teams were equipped with point-of-care tests (POCT), demonstrating the value of POCT in disaster response. ${ }^{15}$

\section{CONCLUSION}

The bacteria and filamentous fungi detected after the East Japan tsunami were widespread in the environment, suggesting the possibility of tsunami lung diagnoses in survivors. That multiple pathogens were detected, as in Case 1, is also suggestive of tsunami lung. These bacteria and fungi are resistant to antimicrobial agents and antifungal agents; therefore, these pathogens are resistant to treatment. 
Because Scedosporium species often attack the central nervous system, and the progression of symptoms is slow, as are respiratory symptoms, careful observation of the central nerve symptom is necessary in such cases. However, both Scedosporium and Legionella pathogens are not specific for tsunami lung, but reported causative agents for pneumonia after near-drowning.

\section{Conflicts of interest}

None declared.

\section{Funding}

None.

\section{References:}

1. Bellos A et al. The burden of acute respiratory infections in crisisaffected populations: a systematic review. Conflict and Health, 2010, 4:3. doi:10.1186/1752-1505-4-3 pmid:20181220

2. Takakura $R$ et al. Follow-up after the Hanshin-Awaji earthquake: diverse influences on pneumonia, bronchial asthma, peptic ulcer and diabetes mellitus. Internal Medicine (Tokyo, Japan), 1997, 36:87-91. doi:10.2169/internalmedicine.36.87 pmid:9099588

3. Jeremijenko A, McLaws ML, Kosasih $\mathrm{H}$. A tsunami related tetanus epidemic in Aceh, Indonesia. Asia-Pacific Journal of Public Health, 2007, 19 Spec No:40-44. pmid:18277527

4. Allworth A. Tsunami lung a necrotizing pneumonia in survivors after the Asian Tsunami. Medical Journal of Australia, 2005, 182 (7):364. pmid:15080423

5. Kao AY et al. Case records of the Massachusetts General Hospital. Case 19-2005. A 17-year-old girl with respiratory distress and hemiparesis after surviving a tsunami. The New England Journal of Medicine, 2005, 352:2628-2636. doi:10.1056/ NEJMcpc059015 pmid:15972870
6. Ebisawa $\mathrm{K}$ et al. Combined Legionella and Escherichia coli lung infection after a tsunami disaster. Internal Medicine (Tokyo, Japan), 2011, 50:2233-2236. doi:10.2169/internalmedicine.50.5800 pmid:21963747

7. Phares $C R$ et al. Epidemiology of severe pneumonia caused by Legionella longbeachae, Mycoplasma pneumoniae, and Chlamydia pneumoniae: 1-year, population-based surveillance for severe pneumonia in Thailand. Clinical Infectious Diseases, 2007. 45:e147-55. doi:10.1086/523003 pmid:18190309

8. Jiva TM et al. Simultaneous legionellosis and invasive aspergillosis in an immunocompetent patient newly treated with corticosteroids. Chest, 1993, 104:1929-1931. doi:10.1378/chest.104.6.1929 pmid:8252995

9. Edelstein PH, Meyer RD. Legionnaires' disease. A review. Chest, 1984, 85:114-120. doi:10.1378/chest.85.1.114 pmid:6360569

10. Katragkou $A$ et al. Scedosporium apiospermum infection after near-drowning. Mycoses, 2007, 50:412-421. doi:10.1111/ j.1439-0507.2007.01388.x pmid:17714363

11. Messori $A$ et al. Mycotic aneurysms as lethal complication of brain pseudallescheriasis in a near-drowned child: a CT demonstration. AJNR, American Journal of Neuroradiology, 2002, 23:16971699. pmid: 12427626

12. Nakamura $Y$ et al. Multiple Scedosporium apiospermum brain abscesses in a woman survivor of a tsunami in northeastern Japan: a case report. Journal of Medical Case Reports, 2011, 5:526. doi: 10.1186/1752-1947-5-526

13. Guarro J et al. Scedosporium apiospermum: changing clinical spectrum of a therapy-refractory opportunist. Medical Mycology, 2006, 44:295-327. doi:10.1080/13693780600752507 pmid: 16772225

14. Thornton CR. Development of an immunochromatographic lateralflow device for rapid serodiagnosis of invasive aspergillosis. Clinical and Vaccine Immunology (CVI), 2008, 15:1095-1105. doi:10.1128/CVI.00068-08 pmid:18463222

15. Kost GJ et al. Katrina, the tsunami, and point-of-care testing: optimizing rapid response diagnosis in disasters. American Journal of Clinical Pathology, 2006, 126:513-520. doi:10.1309/ NWU5E6TOL4PFCBD9 pmid:16938656 